\title{
Socio-Economic Utility of Coastal Flora Growing in and Around Mangrol Taluka (Junagadh) of Gujarat
}

\author{
T. B. Chudasama and S. J. Vyas $\dagger$ \\ Department of Life Sciences, Bhakta Kavi Narsinh Mehta University, Junagadh-362640, Gujarat, India \\ †Corresponding author: S. J. Vyas; vsuhas.13@gmail.com
}

Nat. Env. \& Poll. Tech.

Website: www.neptjournal.com

Received: 03-12-2019

Revised: $14-12-2019$

Accepted: 03-01-2020

Key Words:

Halophytes

Coastal flora

Environmental economics

\begin{abstract}
Halophytes are widely distributed throughout several regions due to the presence of the saline condition. The present paper reflects vegetation cover along with plant species of different kinds in the coastal area of Mangrol taluka of Gujarat, India. It consists of 25 plant species under 25 genera and 12 families of angiosperm. During the field survey, observations were made and plant characteristics and habitat of flora were studied. The main objectives of the present study are the identification, baseline survey and utility of saline plants in the area. The utility of the plants such as ecological and economic (such as medicinal, industrial and commercial, etc.) which can reflect their significance to the society. Majority of the plant species found are naturally occurring, but a few of them are agricultural plants used for various purposes. Due to increasing population growth, urbanization and especially over-demanding medicinal plants people are harvesting without any knowledge of regeneration and conservation, due to which many species are threatened. Because of this, it is very important to conserve plant species, which are extensively utilized in various purposes.
\end{abstract}

\section{INTRODUCTION}

India has a coastline of about 7,516.6 km long with 2.02 million $\mathrm{km}^{2}$ exclusive economic zone and 0.13 million $\mathrm{km}$ 2 continental shelf (Khoshoo 1996) and it covers nine states and two union territories. Gujarat coastal line has a length of approximately $1600 \mathrm{~km}$; it constitutes about $24 \%$ of the total coastal length of India. Coastal zone is an important biogeographically habitats of the Indian subcontinent (Rodgers \& Panwar 1998). Coastal areas are vulnerable to be invaded by tides, and conventional saline-alkali land management measures can hardly achieve the desired results because of serious salinity problems, harsh natural conditions, simple ecosystem structure, poor stability, and fragile ecological environment (Zhang 2018). According to Stoker (1928), the critical level of salinity for plants is $0.5 \%$ of the dry weight. Though the fact that only a small group of higher plants can grow in the saline habitats was recognized many hundred years ago yet the name "halophyte" was assigned to such plants by Pallas in the early nineteenth century. Researches on biological diversity and its values, uses, loss, conservation and management during the last two decades has made a spectacular niche in the field of environmental science (Vyas \& Joshi 2014). In recent years, however, the attention is being paid worldwide to accommodate the salt-tolerant species such as Cressa, Suaeda, Trianthema, Salvadora, to support animal life and providing useful pharmacological as well as economic aspects for human (Nikalje et al. 2018).

The local community around the coast relies on these basic resources, collecting and using many plant species for food, wood, fibre, fuel and medicine. Their high utility in economic and medicinal usage is an important contributing factor to their overexploitation. Salinity is among the major environmental crisis and serious threat to food, fuel and fibre production in the world. This problem, which extends to more than 100 countries, is encountered in all types of climate due to the consequence of both natural processes as well as human interference (Shabala \& Munns 2017). The continual use of coastal plants over many years without any replanting, it has resulted in an accelerated decline in the loss of a wide range of threatened and endangered species. This kind of survey is necessary to explain the usefulness of coastal vegetation to the human community living around the coast.

\section{MATERIALS AND METHODS}

\section{Study Area}

Mangrol taluka of Junagadh district is situated on the extreme coast of Gujarat having dense vegetation of coastal flora. Nine villages of Mangrol taluka and three villages of Madiya taluka were surveyed for the present study $21^{\circ} 13^{\prime} \mathrm{N}$ to $21^{\circ}$ $00^{\prime} \mathrm{N}$ and $69^{\circ} 59^{\prime}$ 'E to $70^{\circ} 13^{\prime} \mathrm{E}$. Total area surveyed under the present study was approximately $40 \mathrm{~km}$, along the coastline 
of Mangrol taluka consisting of various villages on the coast.

\section{Data Collection}

As described earlier, twelve villages were surveyed namely, Antroli, Diwasa, Sangavada, Shil, Lodge, Rahij, Maktupur, Mangrol, Khodada, Khambhadiya, Jujarpur and Chorvad. Plant species along the coast of $40 \mathrm{~km}$ around Mangrol taluka were observed and identified and recorded for the baseline study. The number of each species in all the surveyed villages was recorded along with their habitat in which they were found. Based on the number of species, they were distributed into families in which they belong and utility of all the species were studied.

\section{RESULTS}

In the present investigation a total of 25 species of coastal flora belonging to 18 families were enumerated (Fig. 2) in the coastline of Mangrol taluka (Junagadh). As stated earlier, 12 villages namely Antroli, Diwasa, Sangavada, Shil, Lodge, Rahij, Maktupur, Mangrol, Khodada, Khambhadiya, Jujarpur and Chorvad located on the coastal belt were identified and selected for the current study. From these locations, four species which were found to be dominant in the majority of areas were noted to be Cyprus conglomerates, Halopyrum mucronatum, Prosopis juliflora and Casuarinas equisitifolia. Habitats of these plants are saline and hence can be considered as halo- phytic vegetation. Halophytic vegetation is growing in different parts of the habitat by facing several environmental stresses.

For this study, a maximum of 15 species was found in the village of Lodge, and at least 4 species were found in the village of Shil, in the coast between Antroli to Chorvad village. As shown in Fig. 2, it was interesting to observe and note that the species in the rocky area are higher than in any other habitats such as sand dunes and moist areas.

$\mathrm{SD}=$ Sand dunes, $\mathrm{M}=$ Marshy, $\mathrm{RP}=$ Rock poolsAmong the habitat of selected coastal sites, sand dunes were found to be more followed by rock pools and marshy region. In the coastal areas surrounding Mangrol taluka, 9 villages with sand dunes are given in Table 1 such as Antroli, Sangavada, Shil, Maktupur, Mangrol, Khodada, Khambhadiya, Jujarpur and Chorvad; 2 villages with rocky pool are Lodge and Rahij and 1 village with marshy space is located in Diwasa. According to the survey, highly coastal floras are found on rocky pools (Fig. 2), while vegetation is less common on sandy dunes and marshy places.

As shown in Table 2, the Fabaceae family is found to be most dominant than all other families. There are three types of plants found in the Fabaceae family, namely Lotus garcini, Prosopis juliflora and Indigofera oblingifolia. Two species are found in Convolvulaceae, Poaceae, Acanthaceae and Amaranthaceae family and in addition to this, 1 plant is found in all family found here.

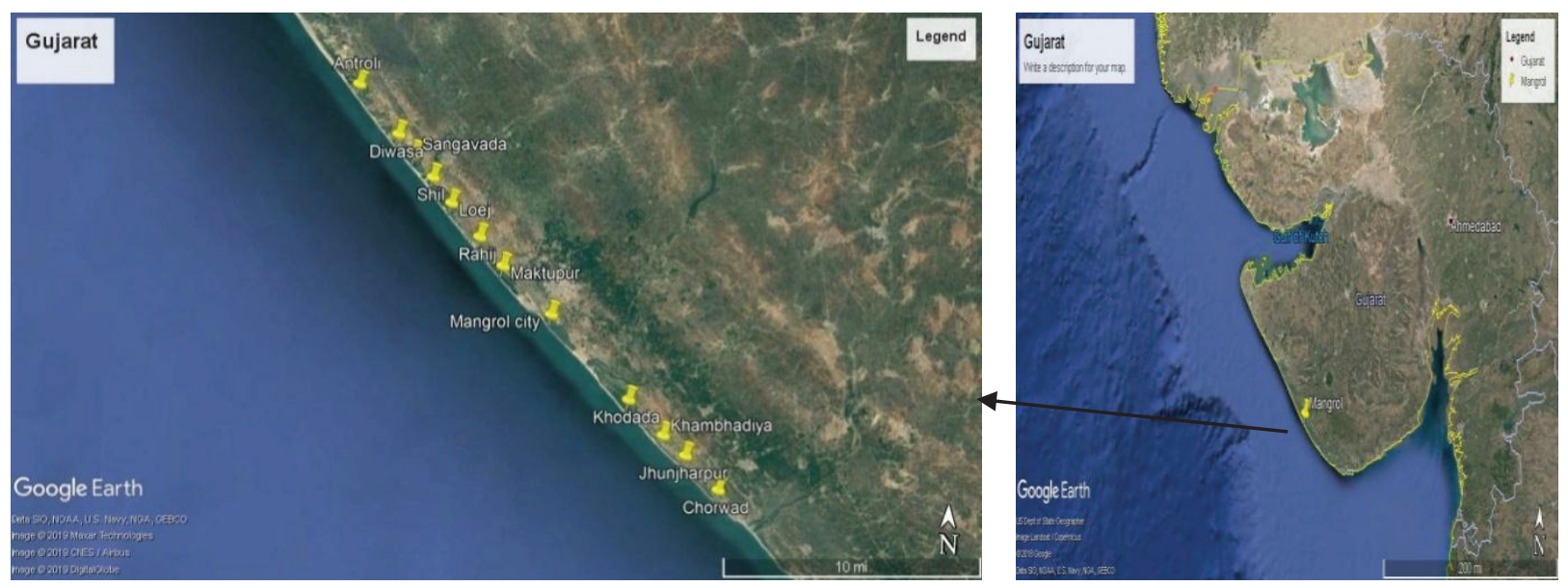

Fig. 1: Map showing study area (Source: Google earth).

Table 1: The habitat in and around Mangrol taluka.

\begin{tabular}{|lll|}
\hline Habitat & No. of habitat & Location \\
\hline SD & 9 & Antroli, Sangavada, Shil, Maktupur, Mangrol, Khodada, Khambhadiya, Jujarpur, Chorvad \\
RP & 2 & Lodge and Rahij \\
M & 1 & Diwasa \\
\hline
\end{tabular}




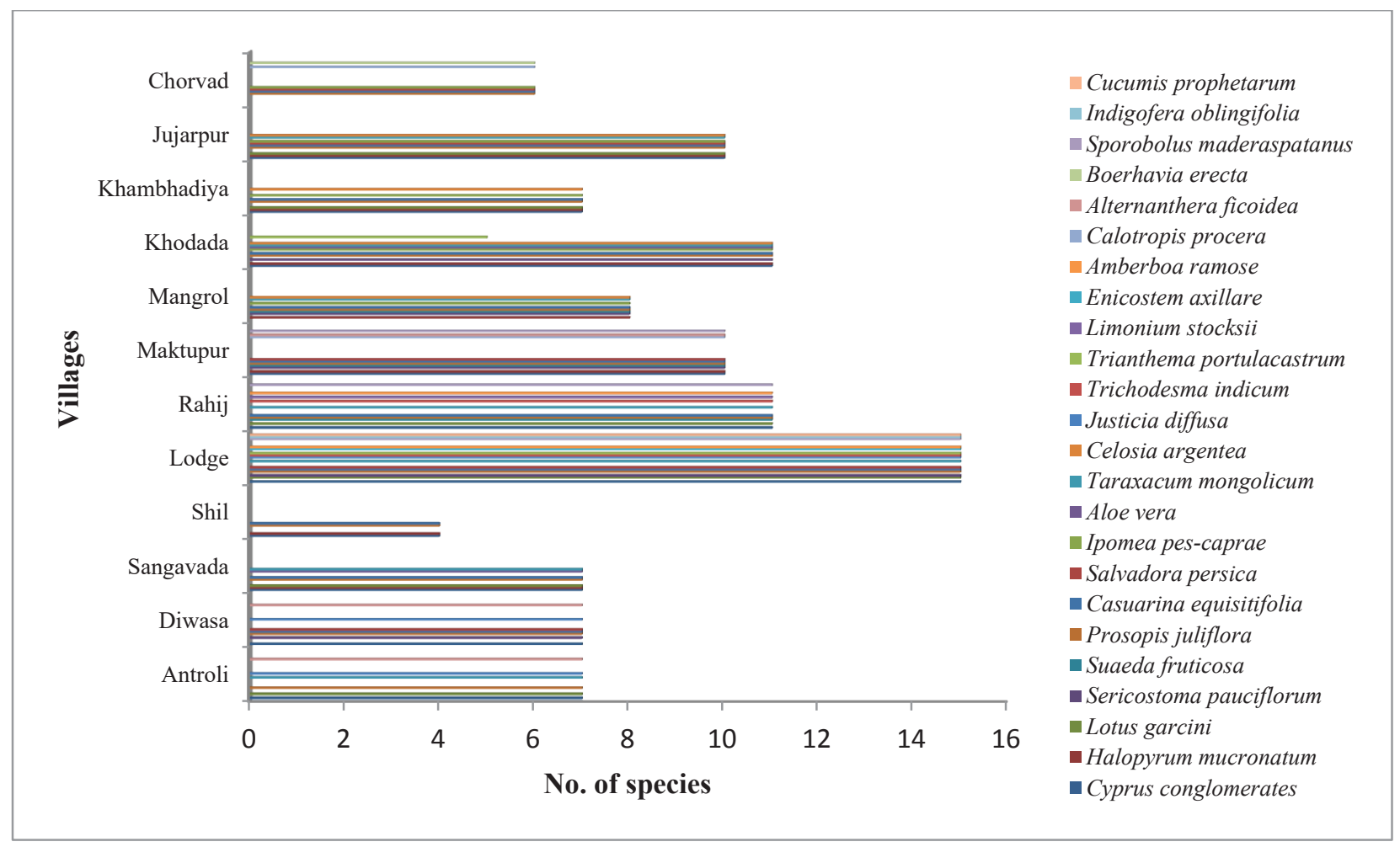

Fig. 2: Village-wise species richness.

Table 2: No. of Genera present in each family.

\begin{tabular}{|c|c|c|}
\hline Sr. No. & Family & No. of Genera \\
\hline 1. & Fabaceae & 3 \\
\hline 2. & Convolvulaceae & 2 \\
\hline 3. & Poaceae & 2 \\
\hline 4. & Acanthaceae & 2 \\
\hline 5. & Amaranthaceae & 2 \\
\hline 6. & Boraginaceae & 1 \\
\hline 7. & Chenopodiaceae & 1 \\
\hline 8. & Cucurbitaceae & 1 \\
\hline 9. & Plumbaginaceae & 1 \\
\hline 10. & Cyperaceae & 1 \\
\hline 11. & Casuarinaceae & 1 \\
\hline 12. & Salvadoraceae & 1 \\
\hline 13. & Asphodelaceae & 1 \\
\hline 14. & Asteraceae & 1 \\
\hline 15. & Aizoaceae & 1 \\
\hline 16. & Gentianaceae & 1 \\
\hline 17. & Apocynaceae & 1 \\
\hline 18. & Nyctaginaceae & 1 \\
\hline
\end{tabular}


Table 3: Checklist of coastal flora showing scientific name, common name, family, habit \& utility.

\begin{tabular}{|c|c|c|c|c|c|c|c|}
\hline $\begin{array}{l}\text { S. } \\
\text { No. }\end{array}$ & Plant name & Family & Habitat & Common name & $\begin{array}{l}\text { Plant part } \\
\text { used }\end{array}$ & Utility & References \\
\hline 1 & Cressa cretica & Convolvulaceae & $\mathrm{H}$ & Rudravanti & $\begin{array}{l}\text { Whole } \\
\text { plant }\end{array}$ & $\begin{array}{l}\text { enriches the blood and useful in } \\
\text { constipation }\end{array}$ & $\begin{array}{l}\text { (Chopra et al. } \\
\text { 2006) }\end{array}$ \\
\hline 2 & $\begin{array}{l}\text { Cyprus } \\
\text { conglomeratus }\end{array}$ & Cyperaceae & $\mathrm{H}$ & - & $\begin{array}{l}\text { Whole } \\
\text { plant }\end{array}$ & used as fodder for animals & (Keblawy 2011) \\
\hline 3 & $\begin{array}{l}\text { Halopyrum } \\
\text { mucronatum }\end{array}$ & Poaceae & G & - & $\begin{array}{l}\text { Whole } \\
\text { plant }\end{array}$ & coastal dune stabilizer & (Khan et al. 1999) \\
\hline 4 & Lotus garcini & Fabaceae & $\mathrm{H}$ & - & Leaves & $\begin{array}{l}\text { Used in benefits of kidney, } \\
\text { controls diarrhea, for sleep } \\
\text { deficiency }\end{array}$ & (Ghulam 2002) \\
\hline 5 & $\begin{array}{l}\text { Sericostoma } \\
\text { pauciflorum }\end{array}$ & Boraginaceae & $\mathrm{S}$ & $\begin{array}{l}\text { Karvash, } \\
\text { Matravalli }\end{array}$ & $\begin{array}{l}\text { Whole } \\
\text { plant }\end{array}$ & $\begin{array}{l}\text { Diabetes, dysentery, urinary } \\
\text { infection }\end{array}$ & (Thakar 1998) \\
\hline 6 & $\begin{array}{l}\text { Suaeda } \\
\text { fruticosa }\end{array}$ & Chenopodiaceae & $\mathrm{S}$ & $\begin{array}{l}\text { Shrubby } \\
\text { seablight }\end{array}$ & $\begin{array}{l}\text { Leaves } \\
\text { Whorl } \\
\text { plant }\end{array}$ & $\begin{array}{l}\text { Treatment of ophthalmia } \\
\text { Making soap and glass }\end{array}$ & (Wichens 2012) \\
\hline 7 & $\begin{array}{l}\text { Prosopis } \\
\text { juliflora }\end{array}$ & Mimosaceae & $\mathrm{T}$ & Gando baval & Wood & $\begin{array}{l}\text { Production of hard wood for } \\
\text { mosaics, boards and sleepers, } \\
\text { good quality firewood }\end{array}$ & (Gomes 1977) \\
\hline 8 & $\begin{array}{l}\text { Casuarina } \\
\text { equisitifoliys }\end{array}$ & Casuarinaceae & $\mathrm{T}$ & Saru, she-oak & $\begin{array}{l}\text { Root, } \\
\text { stem }\end{array}$ & Treatment of dysentery & $\begin{array}{l}\text { (World health } \\
\text { organization 2009) }\end{array}$ \\
\hline 9 & $\begin{array}{l}\text { Salvadora } \\
\text { persica }\end{array}$ & Salvadoraceae & $\mathrm{T}$ & $\begin{array}{l}\text { Meswak tree, } \\
\text { Piludi }\end{array}$ & $\begin{array}{l}\text { Wood } \\
\text { Roots }\end{array}$ & $\begin{array}{l}\text { Useful for fuel } \\
\text { Used as a toothbrushes }\end{array}$ & (Uphof 1959) \\
\hline 10 & $\begin{array}{l}\text { Ipomea pes- } \\
\text { caprae }\end{array}$ & Convolvulaceae & $\mathrm{C}$ & $\begin{array}{l}\text { Morning glory } \\
\text { or goat's foot, } \\
\text { Maryada vel }\end{array}$ & $\begin{array}{l}\text { Stem } \\
\text { Leaves }\end{array}$ & $\begin{array}{l}\text { Made in ropes } \\
\text { Used to extirpate fungoid } \\
\text { growth of ulcers } \\
\text { Relief in bladder diseases }\end{array}$ & (Burkill 1985) \\
\hline & & & & & Root & & \\
\hline 11 & Aloe vera & Asphodelaceae & $\mathrm{H}$ & $\begin{array}{l}\text { Indian aloe, } \\
\text { Kuvarpathu }\end{array}$ & $\begin{array}{l}\text { Whole } \\
\text { plant }\end{array}$ & $\begin{array}{l}\text { Cosmetic \& medicinal purpose, } \\
\text { Used in commercially as } \\
\text { an ingredient in yogurts, } \\
\text { beverages, and some desserts }\end{array}$ & $\begin{array}{l}\text { (Reynolds \& Tom } \\
\text { (Ed.) 2004) }\end{array}$ \\
\hline 12 & $\begin{array}{l}\text { Taraxacum } \\
\text { mongolicum }\end{array}$ & Asteraceae & $\mathrm{H}$ & Dandelions & $\begin{array}{l}\text { Whole } \\
\text { plant }\end{array}$ & $\begin{array}{l}\text { Treat inflammation, swollen } \\
\text { lymph nodes, cysts and } \\
\text { abscesses, as well detoxifying } \\
\text { the kidney and liver }\end{array}$ & $\begin{array}{l}\text { (Roger \& George } \\
\text { 2004) }\end{array}$ \\
\hline 13 & $\begin{array}{l}\text { Cucumis } \\
\text { prophetarum }\end{array}$ & Cucurbetaceae & $\mathrm{C}$ & Spiked melon & Fruit & Used as an emetic & (Uphof 1959) \\
\hline 14 & Justicia diffusa & Acanthaceae & $\mathrm{H}$ & Water willow & Leaves & Used in ophthalmic & $\begin{array}{l}\text { (Chopra et al. } \\
\text { 1986) }\end{array}$ \\
\hline 15 & $\begin{array}{l}\text { Trichodesma } \\
\text { indicum }\end{array}$ & Boraginaceae & $\mathrm{H}$ & $\begin{array}{l}\text { Undhaphuli, } \\
\text { Indian borage }\end{array}$ & $\begin{array}{l}\text { Leaves, } \\
\text { root }\end{array}$ & treatment of cough & $\begin{array}{l}\text { (Subban \& } \\
\text { AlarmalMangai } \\
\text { 2012) }\end{array}$ \\
\hline 16 & $\begin{array}{l}\text { Trianthema } \\
\text { portulacastrum }\end{array}$ & Aizoaceae & $\mathrm{H}$ & Black pigweed & $\begin{array}{l}\text { Whole } \\
\text { plant }\end{array}$ & $\begin{array}{l}\text { Used as a vermifuge and is } \\
\text { useful in rheumatism }\end{array}$ & $\begin{array}{l}\text { (Aggarwal \& Kaur } \\
\text { 2017) }\end{array}$ \\
\hline 17 & $\begin{array}{l}\text { Limonium } \\
\text { stocksii }\end{array}$ & Plumbaginaceae & $\mathrm{H}$ & Sea-lavender & $\begin{array}{l}\text { Whole } \\
\text { plant }\end{array}$ & $\begin{array}{l}\text { Used as a food, pharmaceutical, } \\
\text { cosmetics and other industrial } \\
\text { products }\end{array}$ & $\begin{array}{l}\text { (Akashi \& Ayabe } \\
\text { 2010) }\end{array}$ \\
\hline 18 & $\begin{array}{l}\text { Enicostem } \\
\text { axillare }\end{array}$ & Gentianaceae & $\mathrm{H}$ & Mahmejo & $\begin{array}{l}\text { Whole } \\
\text { plant }\end{array}$ & $\begin{array}{l}\text { Used as a laxative, stomachic } \\
\text { and tonic }\end{array}$ & $\begin{array}{l}\text { (Chopra et al. } \\
\text { 1986) }\end{array}$ \\
\hline
\end{tabular}




\begin{tabular}{|c|c|c|c|c|c|c|c|}
\hline $\begin{array}{l}\text { S. } \\
\text { No. }\end{array}$ & Plant name & Family & Habitat & Common name & $\begin{array}{l}\text { Plant part } \\
\text { used }\end{array}$ & Utility & References \\
\hline 19 & $\begin{array}{l}\text { Lapidagathis } \\
\text { cristata }\end{array}$ & Acanthaceae & $\mathrm{H}$ & $\begin{array}{l}\text { Hiran-chaaro } \\
\text { (pathar-kotar) }\end{array}$ & $\begin{array}{l}\text { Whole } \\
\text { plant }\end{array}$ & $\begin{array}{l}\text { Antiallergic medicine } \& \text { used } \\
\text { as a fodder }\end{array}$ & (Panda 2002) \\
\hline 20 & $\begin{array}{l}\text { Calotropis } \\
\text { procera }\end{array}$ & Apocynaceae & $\mathrm{S}$ & Auricula tree & $\begin{array}{l}\text { Stem } \\
\text { Root } \\
\text { Seed } \\
\text { capsules } \\
\text { Leaves }\end{array}$ & $\begin{array}{l}\text { Termite proof stem used for } \\
\text { roofing and building huts } \\
\text { Treatment of snakebites } \\
\text { Used as a stuffing material in } \\
\text { mattresses } \\
\text { Treatment of asthma }\end{array}$ & $\begin{array}{l}\text { (Von Maydell } \\
\text { 1990) }\end{array}$ \\
\hline 21 & $\begin{array}{l}\text { Alternanthera } \\
\text { ficoidea }\end{array}$ & Amaranthaceae & $\mathrm{H}$ & Calico plant & $\begin{array}{l}\text { Whole } \\
\text { plant }\end{array}$ & Bioagent in greywater treatment & $\begin{array}{l}\text { (Abbasi \& Tauseef } \\
\text { 2018) }\end{array}$ \\
\hline 22 & $\begin{array}{l}\text { Boerhavia } \\
\text { erecta }\end{array}$ & Nyctaginaceae & $\mathrm{H}$ & $\begin{array}{l}\text { Erect } \\
\text { spiderling, } \\
\text { Satodi }\end{array}$ & $\begin{array}{l}\text { Whole } \\
\text { plant }\end{array}$ & Used as a vermifuge & $\begin{array}{l}\text { Achigan-dako } \\
\text { 2009) }\end{array}$ \\
\hline 23 & $\begin{array}{l}\text { Sporobolus } \\
\text { maderaspatanus }\end{array}$ & Poaceae & G & - & $\begin{array}{l}\text { Whole } \\
\text { plant }\end{array}$ & Used as a forage & (Joshi 2011) \\
\hline 24 & $\begin{array}{l}\text { Celosia } \\
\text { argentea }\end{array}$ & Amaranthaceae & $\mathrm{H}$ & $\begin{array}{l}\text { Common } \\
\text { cockscomb }\end{array}$ & $\begin{array}{l}\text { Whole } \\
\text { plant }\end{array}$ & $\begin{array}{l}\text { It could purge the liver of } \\
\text { pathogenic fire, improve } \\
\text { eyesight, and eliminate } \\
\text { nephelium }\end{array}$ & (Shen 1997) \\
\hline 25 & $\begin{array}{l}\text { Indigofera } \\
\text { oblingifolia }\end{array}$ & Fabaceae & $\mathrm{S}$ & Jhil, Jhiladi & $\begin{array}{l}\text { Leaves } \\
\text { Stem } \\
\text { Root }\end{array}$ & $\begin{array}{l}\text { Treat skin rash \& stomach pain } \\
\text { Treatment of mercurial } \\
\text { salivation } \\
\text { Used as a purgative }\end{array}$ & $\begin{array}{l}\text { (Chopra et al. } \\
1986)\end{array}$ \\
\hline
\end{tabular}

$\mathrm{H}=$ herb, $\mathrm{S}=$ shrub, $\mathrm{T}=$ tree and $\mathrm{G}=$ grass and $\mathrm{C}=$ Climber

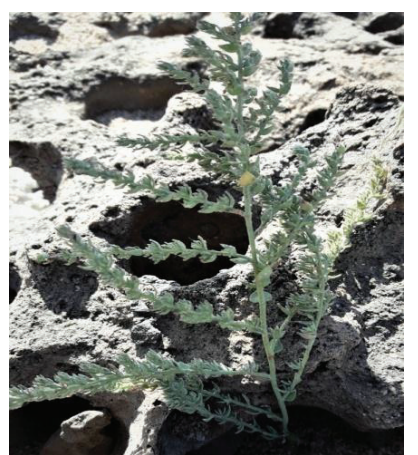

1: Cressa cretica

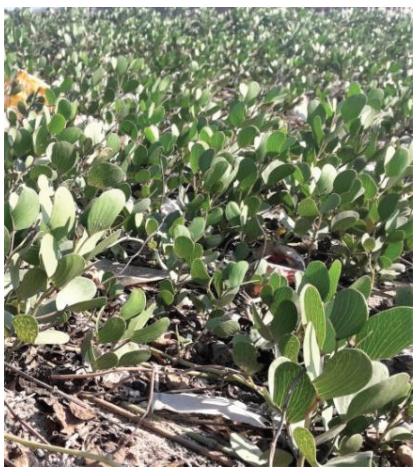

4: Ipomea pes-caprae

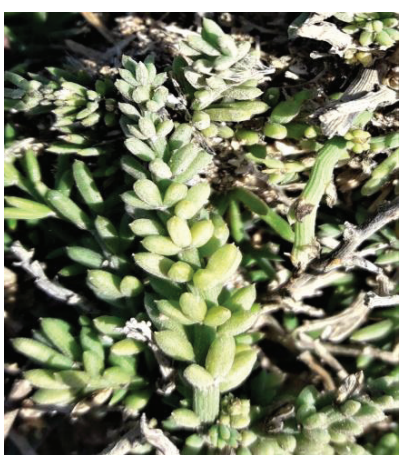

2: Suaeda fruticosa

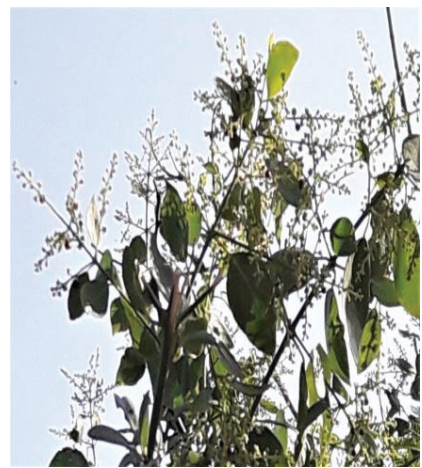

5: Salvadora persica

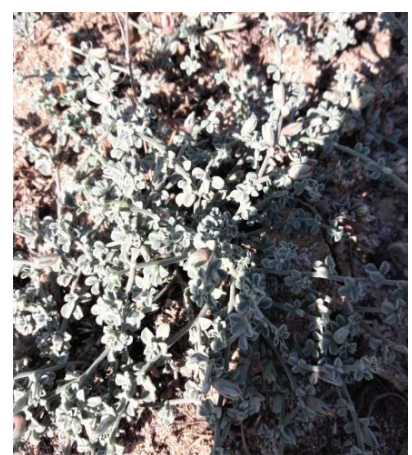

3: Sericostoma pauciflorum

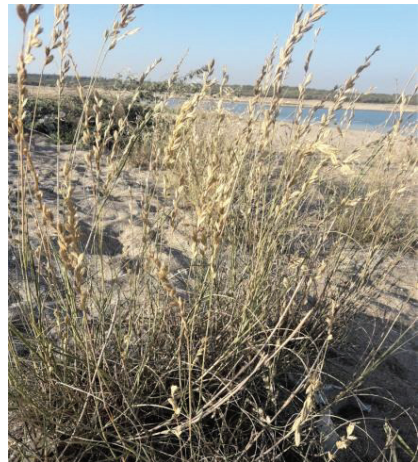

6: Halopyrum mucronatum 


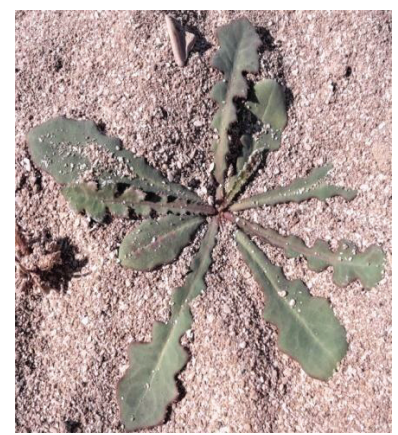

7: Taraxacum mongolicum

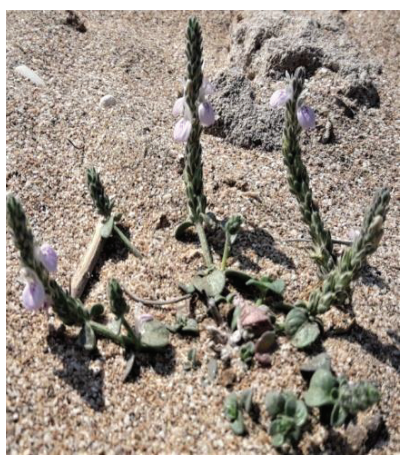

10: Justicia diffusa

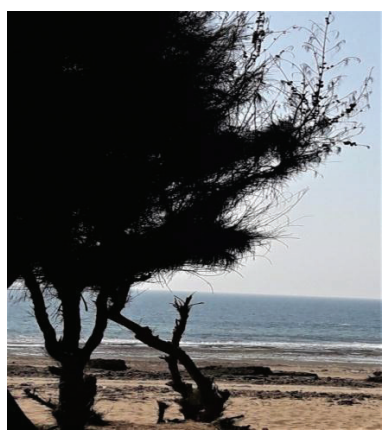

13: Casuarinas equisitifoliys

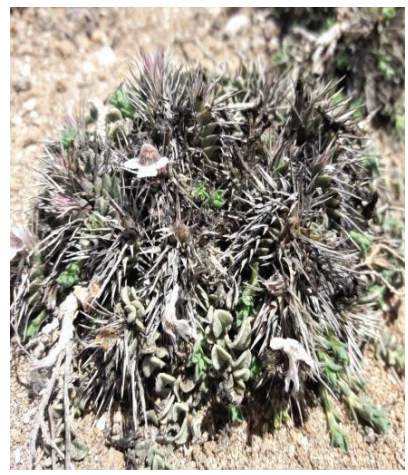

16: Amberboa ramose

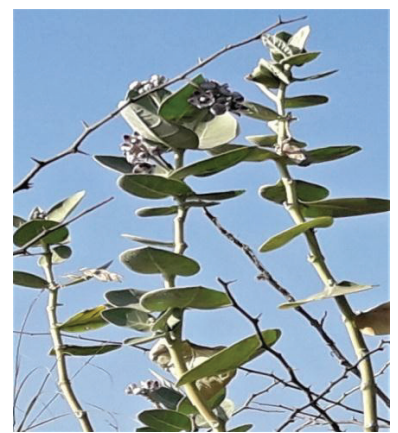

8: Calotropis procera

PLATE 1

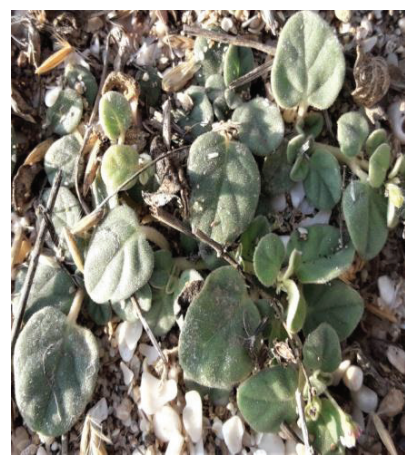

11: Trianthema portulacastrum

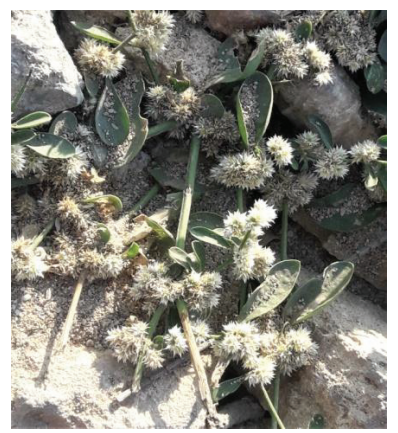

14: Alternanthera ficoidea

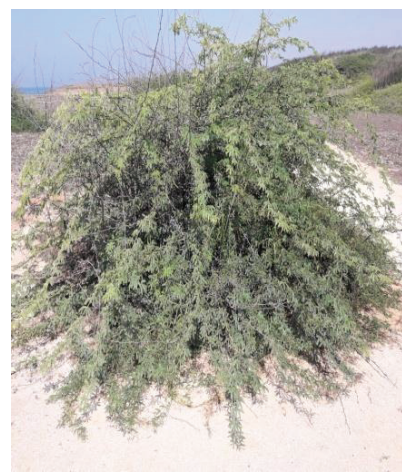

17: Prosopis juliflora

PLATE 2

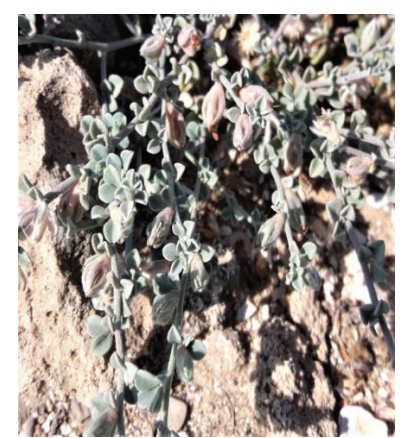

9: Lotus garcini

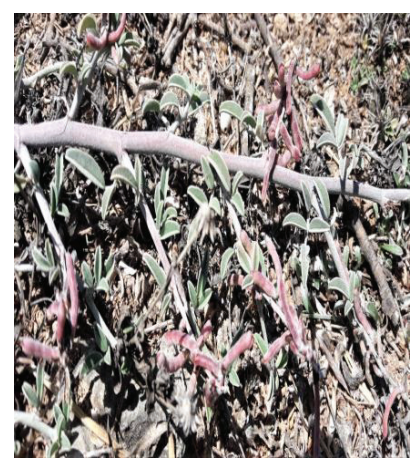

12: Indigofera oblingifolia

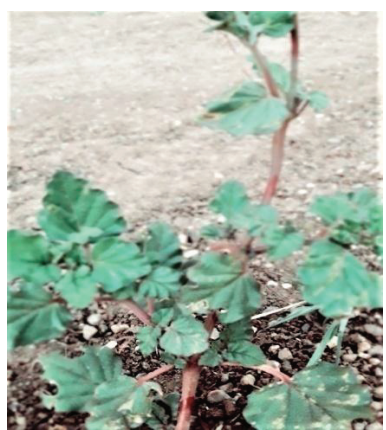

15: Boerhavia erecta

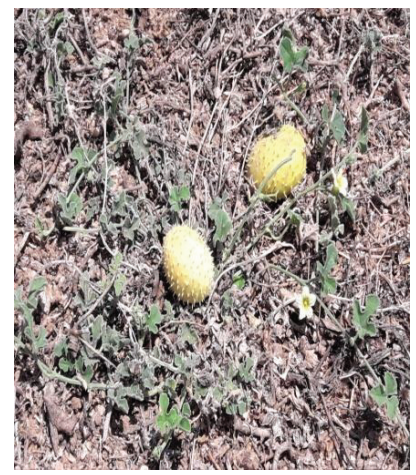

18: Cucumis prophetarum 


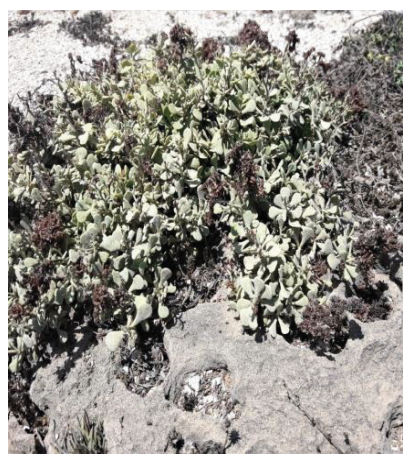

19: Limonium stocksii

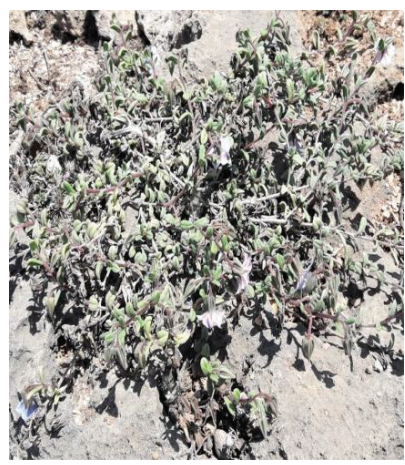

22: Trichodesma indicum

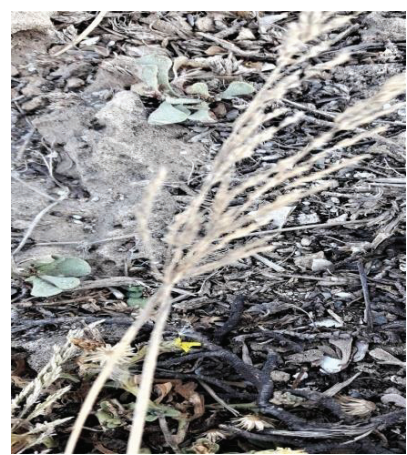

20: Sporobolus maderaspatanus

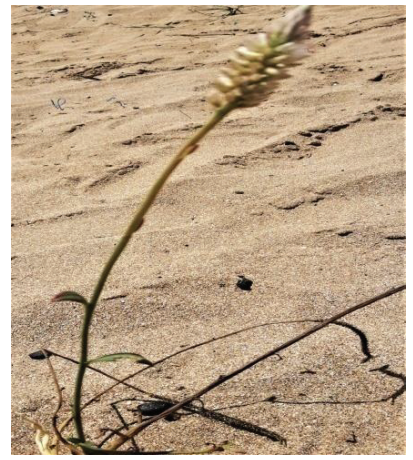

23: Celosia argentea

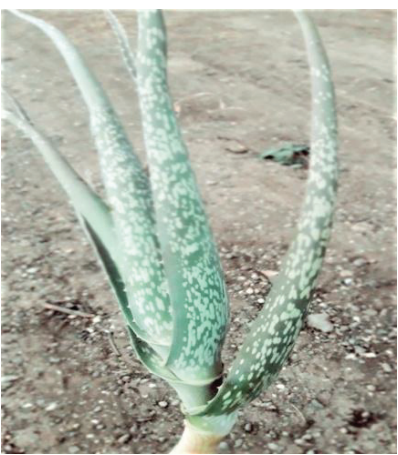

21: Aloe vera

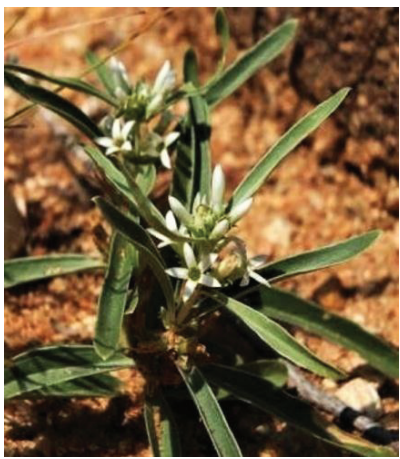

24: Enicostem axillare

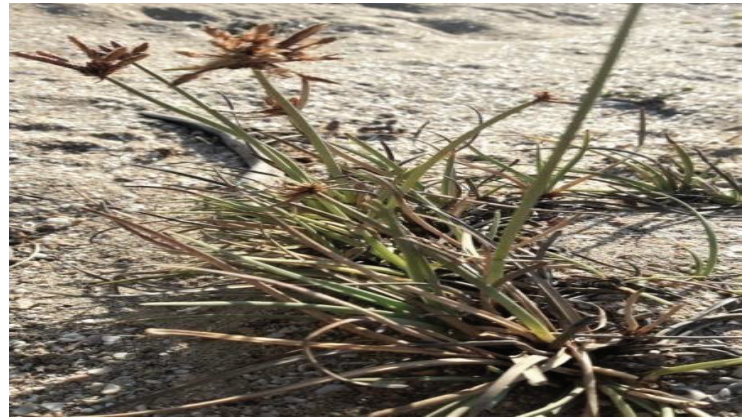

25: Cyprus conglomerates

PLATE 3

Fig. 3: Plant species of the study area (Plates 1-3).

Coastal vegetation is not commonly used for economic gain, but it also identified for ethnomedicinal, handicrafts and many other uses. Numerous reports have documented the utility of coastal flora in rural and tribal areas all over the world as a successful home remedy against different ailments (Hamburger \& Hostettmann 1991, Hammiche \& Maiza 2006, Hussain et al. 2003). Among the species, herbs were found to be more (14 sp.) followed by shrubs ( $4 \mathrm{sp}$.), trees ( $3 \mathrm{sp}$.), grass $(2 \mathrm{sp}$.) and climbers (2 sp.) (Fig. 3). In the available literature, the vegetation of coastal flora was highly used in traditional and ethnobotanical interests were recorded after the critical screening.

\section{CONCLUSION}

The present work reflected a detailed and comprehensive database and descriptive survey of coastal flora which compiled the economic and ecological utility of the plant species observed for identifying the potential of the Mangrol coast. Throughout the $40 \mathrm{~km}$ coastline of the study area, twenty-five 
coastal species were observed and recorded for their utility. The interesting fact is to interpret that these 25 species were listed under 18 families, which showed the immense potential of all the species observed in various treatments of diseases. It also revealed that Mangrol taluka having a coastal belt in highly diversified in terms of plant species with reference to the coastal line based on the habitat. These plant species were recorded on different coastal habitats comprising of sandy, rocky and marshy.

\section{ACKNOWLEDGMENT}

The authors would like to thanks Department of Forest, Development \& Management, Gujarat state for providing the permission to carry out this research.

\section{REFERENCES}

Abbasi, S.N. and Tauseef, S.M. 2018. Use of terrestrial weed Alternanthera ficoidea in treating greywater treatment in soil-less SHEFROL bioreactor. Water Sci. Technol., 77(8): 2005-2013.

Achigan-dako, E. 2009. Traditional Vegetables in Benin, Darwin initiative. International Foundation for Science, 2009.

Akashi, T. and Ayabe, S. 2010. Comprehensive Natural Products. Elsevier Science, Vol. 2

Burkill, H.M., 1985. The Useful Plants of West Tropical Africa Royal Botanic Gardens. London, 3, pp.101.

Chopra, R. N., Nayar, S. L. and Chopra, I. C. 1986. Glossary of Indian Medicinal Plants. Council of Scientific and Industrial Research, New Delhi.

Chopra, R.N., Nayar, S.L. and Chopra, I.C. 2006. Glossary of Indian Medicinal Plants. National Institute of Science Communication and Information Resources, New Delhi, pp. 80.

Ghulam, S. 2002. The Phytochemical and Phytopharmacological studies on Saraca indica, Capparis decidua and Lotus garcinia. Deptt. of Pharmacognosy, University of Karachi, Pakistan.

Gomes, P.A. 1977. Forragens Fartas Na Seca. 4th Ed. São Paulo, Nobel, pp. 223.

Hamburger, M. and Hostettmann, K. 1991. Bioactivity in plants: The link between phytochemistry and medicine. Phytochemistry, 30: 3864-3874.

Hammiche, V. and Maiza, K. 2006. Traditional medicine in Central Sahara: Pharmacopoeia of Tassili N'ajjer. Journal of Ethnopharmacology, 105: 358-367.

Hussain, N. A., Naseem, R., Sarwar, G., Mujeeb, F. and Jamil, M. 2003. Domestication/cultivation scope of medicinal crops on salt-affected soils. Conservation and Sustainable Uses of Medicinal and Aromatic Plants of Pakistan, 2-4: 37-43.

Joshi, A.J. 2011. Monograph on Indian halophytes. Ocean \& Atmo. Sci. and Techno. Cell, 70-73.
Kaur, M. and Aggarwal, N.K. 2017. Trianthema portulacastrum L.- The noxious weed and its control. Adv. Plants Agric. Res., 6(3): 62-64.

Keblawy, A., Neyadi, S.S., Rao, M.V. and Marzouqi, A.H. 2011. Interactive effects of salinity, light and temperature on seed germination of sand dunes glycophyte Cyprus conglomeratus growing in the United Arab Emirates deserts. Seed Sci. \& Technol., 39: 364-376.

Khan, M.A., Ungar, I.A. and Showalter, A.M. 1999. Effects of salinity on growth, ion content and osmotic relations in Halopyrum mucronatum (L). Stapf. J. Plant Nutr., 22: 191-204.

Khoshoo, T.N. 1996. Vesicular-arbuscular mycorrhizae of Hawaiian dune plants. Curr. Sci., 71: 506-513.

Nikalje, G.C., Srivastava, A.K., Pandey, G.K. and Suprasanna, P. 2018. Halophytes in biosaline agriculture: Mechanism, utilization, and value addition. Land Degradation and Development, 29(4): 1081-1095.

Panda, H. 2002. Medicinal Plants Cultivation and Their Uses. National Institute of Industrial Research.

Reynolds, T. and Tom (Ed.) 2004. Aloes: The genus Aloe (Medicinal and Aromatic Plants- Industrial Profiles. CRC Press.

Rodgers, W.A. and Panwar, H.S. 1998. Planning a Wildlife Protected Area Network in India. Wildlife Institute of India, Dehra Dun, India, Vol. 1.

Roger, P. and George, D. 2004. Encyclopedia of Medicinal Plants 1: Education and Health Library, pp. 398.

Shabala, S. and Munns, R. 2017. Salinity stress: physiological constraints and adaptive mechanisms. In: Shabala, S. (ed.) Plant Stress Physiology. 2nd edn. CABI, Wallingford. (2017): 24-63.

Shen, N. 1997. Shen Nong Ben Cao. Liaoning Science \& Technology Press, Shenyang, pp. 40

Stoker, O. 1928. Das halophytes problem. Erg. Biol., 3: 265-353.

Subban, R. and Alarmal Mangai, S. 2012. Comparative corrosion inhibition effect of imidazole compounds of Trichodesma indicum (Linn) R. Br. on C38 Steel in $1 \mathrm{M} \mathrm{HCl}$ medium. Journal of Chemistry, 2012, 2013:1-4.

Thakar, J.K. 1998. Vanaspati Shashtra, $2^{\text {nd }}$ edition. Pravin Prakashan, Rajkot, pp. 471-480.

Uphof. J. C. 1959. Dictionary of Economic Plants. Weinheim.

Von Maydell, H. 1990. Trees and Shrubs of the Sahel. Their characteristics and Uses. Deutsche Gesellschaft Technische usammenarbeit, Germany.

Vyas, S.J. and Joshi, A.J. 2014. Mineral composition in leaves of some halophytic species of 'Bhal' region in Gujarat. Research Journal of Biology, 2: 99-103.

Wichens, G.E., David, V. and Goodin, R. 2012. Plants for arid lands. In: Proceedings of the Kew International Conference on Economic Plants for Arid Lands held in the Joderell Laboratory, Royal Botanic Gardens, Kew, England, 23-27 July 1984.

WHO 2009. Medicinal Plants in Papua, New Guinea, World Health Organisation.

Zhang, G., Wang, C., Yang, H., Zhou, Z., Zhang, Y. and Zhao, L. 2018. Experimental research on improving the salt tolerance of plants in coastal saline soil - A case study of Huanghua City in Hebei Province of China. Nat. Env. \& Poll. Tech., 17(2): 459-468. 